\title{
Trabajo epigráfico en la tumba de Hery (TT 12)
}

Gemma MENÉNDEZ GÓMEZ

El objetivo de este artículo es exponer el trabajo epigráfico que está realizando la misión hispano-egipcia en las tumbas de Djehuty (TT 11) y Hery (TT 12), centrando nuestra atención en esta última. Esta labor comprende tres tareas diferentes: en primer lugar, documentar el estado actual de la pared de la tumba y su decoración; en segundo lugar, registrar y estudiar los fragmentos de relieve hallados en los trabajos de excavación; y en último lugar, recopilar la documentación epigráfica previa a nuestro trabajo, donde juegan un papel fundamental los squeezes de Spiegelberg conservados en el Griffith Institute de Oxford. Todo ello, junto al estudio de los paralelos iconográficos de las escenas e inscripciones, permite reconstruir parcialmente las lagunas decorativas que el paso del tiempo ha dejado en las paredes de la tumba.

\section{Epigraphical work in the tomb of Hery (TT 12)}

The aim of this article is to describe the epigraphic work that the Spanish-Egyptian Mission is carrying out in the tombs of Djehuty (TT 11) and Hery (TT 12), focusing attention on the latter. This work covers three different tasks: firstly, recording the current state of the wall and its decoration; secondly, registering and studying the fragments of relief that were found during the excavation; and finally, collecting the previous epigraphic documentation, where the squeezes of Spiegelberg, kept in the Griffith Institute in Oxford, play an important role. These tasks, together with the study of iconographic parallels of the scenes and inscriptions, enable us to reconstruct the decorative gaps which have resulted from the passing of time.

KeY wORDs: Thebes, 18th Dynasty, Hery, Spiegelberg, epigraphic methodology

$\mathrm{L}$ a tumba de Hery (TT 12) ${ }^{1}$ se encuentra ubicada en la necrópolis de Dra Abu el-Naga norte, lugar que fue utilizado como cementerio nobiliario desde el Reino Medio hasta el Segundo Periodo Intermedio, cuando se convirtió en necrópolis real ${ }^{2}$. Su importancia despertó un gran interés funerario a partir del Reino Nuevo, provocando la reutilización de muchas tumbas anteriores y la excavación de pozos de enterramiento convirtiéndola en una de las necrópolis con mayor ocupación.

Hery fue funcionario de Ahmose y Amenhotep I ejerciendo los cargos de "escriba- $w b$ " " y "su-

* Mi agradecimiento a José Manuel Galán y Andrés Diego Espinel que han tomado parte en la labor epigráfica de los relieves de la tumba, la catalogación de los fragmentos y la investigación llevada a cabo en el Griffith Institute de Oxford.

1. PM I (1): 24-25; Kampp, 1996, I: 191-192; Galán, 2006: 779, 782, fig. 2.

2. Los hallazgos del ajuar funerario de Ahhotep, entre otros, aportaron indicios sobre la localización de otras tumbas reales de la dinastía XVII, algunas de las cuales actualmente se han perdido. Winlock 1924; Dewatcher, 1985.

Fecha de recepción: 15 de noviembre de 2006

Fecha de aceptación: 25 de junio de 2007 
pervisor del doble granero de la esposa real y madre del rey Ahhotep"4. Su madre juega un papel primordial en las inscripciones de su tumba. Esta mujer llamada Ahmose, tiene el título de hrw-

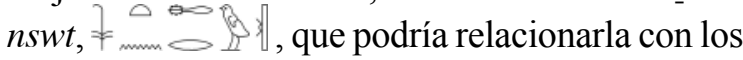
miembros de la corte o del entorno real ${ }^{5}$. El resto de sus familiares son nombrados uno por uno en la escena de banquete: tres hermanos, diez hermanas, una hermanastra, cinco hijos y su esposa, cuyo nombre no se ha conservado. En las inscripciones de las paredes no hay ninguna mención al padre de Hery. La falta de representaciones de la figura paterna en las tumbas de la dinastía XVIII podría estar ligada al alto estatus de la madre del difunto, teniendo ésta preeminencia respecto al resto de las figuras ${ }^{6}$. En el caso de que el esposo fuera de un estatus inferior a la mujer, tal vez no hubiera resultado conveniente que apareciera en un segundo plano y se optó por no representarle?

La entrada original de la tumba fue tapiada por el Servicio de Antigüedades a principios del siglo $\mathrm{XX}^{8}$. La tumba posee otro acceso a través de un agujero que se abre en la pared este de la sala transversal de la tumba de Djehuty (TT 11) y de la -399-, y que rompe parte de la pared oeste del pasillo de Hery ${ }^{10}$.
La planta de la tumba consiste en un estrecho pasillo que conduce a una sala rectangular, algo irregular, con un grueso pilar central. En el centro, junto al pilar, un agujero en el techo ha provocado la colmatación de la sala por la caída de escombros. La decoración sobre la que estamos trabajando se encuentra en el pasillo de la tumba, ya que los escombros de la sala interior no nos permiten ver si sus paredes están decoradas. Una vez que estos sean retirados, comprobaremos si han actuado como protectores de los relieves que podrían estar cubriendo ${ }^{11}$.

La longitud de los paneles del pasillo es de unos 6'35 m de largo y alrededor de 1'95 de alto. Se trata de escenas realizadas en bajorrelieve que no han conservado restos de pintura. El estilo clásico de los relieves es de influencia del Reino Medio, tal y como se observa en la musculatura de las piernas $\mathrm{y}$ en los rostros de las figuras. Esta tendencia de imitar el arte de la dinastía XII fue bastante común a comienzos de la XVIII ${ }^{12}$. Las escenas están claramente diferenciadas, reuniendo toda la temática iconográfica característica del Reino Nuevo ${ }^{13}$ : la procesión de ofrendas, las escenas de caza y de pesca, la peregrinación a Abidos, el cortejo fúnebre, la escena de banquete y la lista de ofrendas.

3. Wb. I: 292, traduce $w b 3$ como "Diener", “Aufwärter".

4. Bohleke, 2000: 52-53, ofrece una lista de todos los personajes del Reino Nuevo que portaron este título. Según el autor, Hery es uno de los ejemplos más significativos que muestran los cambios que se produjeron en este periodo. Tras la reconquista del poder por parte de Ahmose, y debido a la necesidad de reforzar la organización de un territorio que había estado dividido durante casi 150 años, nombró a los oficiales tebanos de confianza como responsables de esta labor antes que a los recién conquistados del norte.

[ 110 ] 5. Se trata de otro hápax. Whale, 1989: 12, sugiere una conexión real.

6. Nims, 1980: 25, n. 55.

7. Sobre la ausencia de la figura del esposo en monumentos funerarios femeninos véase, Roth, 1999.

8. Gardiner y Weigall, 1913: 16-17. Se calcula que la entrada fue tapiada entre 1910-1912 para frenar el saqueo que habían sufrido los relieves. De la misma forma se actuó en la tumba contigua de Djehuty, cuyo patio fue fotografiado antes de que fuera techado y cerrado, ver Gardiner MSS 28.651 y 28.652; Griffith Institute $\mathrm{n}^{\mathrm{o}} 1684 ; 1731$.

9. Kampp, 1996, I: 191; II: 768; Galán, 2006: 782, fig. 2.

10. Este acceso se debió realizar no más tarde del siglo $\mathrm{V}$ d. C., como demuestran los restos de un grafito demótico en uno de los laterales del agujero de paso.

11. Algo similar ocurre en la tumba de Djehuty. Los trabajos de excavación dentro de la capilla han demostrado que los escombros que la colmataban han protegido la decoración.

12. Romano, 1976; Romano, 1983. 
Hay tres tipos de inscripciones distribuidas en las paredes:

- Dos frisos de texto con la fórmula htp-di-nsw que coronan ambas paredes del pasillo.

- Inscripciones incisas, de menor tamaño y menos elaboradas, insertadas junto a las figuras, que hacen referencia a los nombres propios de los familiares del difunto o a los títulos de los sacerdotes involucrados en la lista de ofrendas.

- Grafitos demóticos pintados en rojo que se reparten de forma dispar en la pared oeste y que son testimonio de una posterior reutilización de la tumba como lugar de enterramiento de momias de ibis y halcones ${ }^{14}$.

\section{Egiptólogos en la tumba de Hery}

Una de las primeras personas cuyo testimonio nos ha llegado de su visita a Hery fue JeanFrançois Champollion. Acompañado por Ippolito Rosellini, entró en $1829^{15}$ por la tumba adyacente de $\mathrm{Baki}^{16}$, acceso que actualmente está bloqueado con tierra y piedras, pasando por alto la TT 11, cuyo acceso se encuentra hoy abierto pero que entonces debía de estar tapado. Su labor allí consistió en tomar nota de algunas de las inscripciones, sobre todo de las que ocupaban la pared oeste, que se publicaron en sus Notices Descriptives ${ }^{17}$. Los cuadernos de campo de Rosellini se conservan inéditos en la biblioteca de la Universidad de Pisa $^{18}$.
Casi 15 años después, el 5 de diciembre de 1844, Richard Lepsius entró en la tumba de Hery. Copió parte de las inscripciones y de los nombres que aparecían en la escena de banquete. Sin embargo, no todas sus notas de la tumba fueron publicadas en el volumen III del Denkmäler ${ }^{19}$, pues uno de sus cuadernos permaneció extraviado por unos años. La información que recopiló se puede ver completa en su Diario, que actualmente se conserva en la Academia de Ciencias de Berlín ${ }^{20}$.

El visitante más importante para nuestro trabajo epigráfico fue Wilhelm Spiegelberg, que visitó la tumba en el invierno de 1896. Interesado tanto por las inscripciones como por los relieves de la TT 11 y de la TT 12, realizó una serie de estampados o squeezes de ambas tumbas. Spiegelberg calcó la estela autobiográfica de Djehuty que se conoce como "estela de Northampton"21 y la pared oeste de la tumba de Hery, donde está representada la procesión funeraria y el banquete, dejando a un lado la pared este, que debía encontrarse ya por entonces muy deteriorada. En la actualidad, estos squeezes se conservan en el archivo del Griffith Institute, y se han convertido en un documento de gran valor epigráfico ya que a través de ellos podemos ver los cambios que ha sufrido la pared desde 1896 hasta hoy y conocer las partes de los relieves que han desaparecido. Durante su visita, Spiegelberg comprobó el valor arqueológico de la zona y, pocos años después, decidió excavar en Dra Abu el-Naga junto a Percy Newberry, gracias a la financiación del Marqués

13. Un cuadro ilustrativo respecto a la ubicación de estas escenas se puede ver en Menéndez, 2005: 50, fig. 1.

14. Marquis of Northampton, Spiegelberg y Newberry, 1908: 19-25, láms. XXVI-XXX; Strudwick, $2003: 172$.

15. No sabemos la fecha exacta en la que ambos visitaron la tumba, pero la estancia en Luxor de Rosellini duró del 8 de marzo hasta el mes de agosto.

16. Esta tumba no está numerada y no debe confundirse con la TT 18, PM I (1): 32, cuyo propietario también se llama Baki. La única documentación disponible se puede encontrar en Serrano, 2005; Galán, 2006: 782, fig. $2,784$.

17. Champollion, 1873: 543-544.

18. Rosellini MSS. 284.G 61.

19. Lepsius, 1897-1913, III: 238.

20. Diario de Lepsius, del 30 de octubre al 7 de diciembre, $n^{\circ}$ 77-78: 421-423. 
de Northampton. La campaña duró unos cuatro meses, del 7 de noviembre de 1898 al 27 de enero de $1899^{22}$. El trabajo de excavación y de documentación quedó a cargo de Spiegelberg, quien anotó todos los descubrimientos en un diario que también se conserva en el Griffith Institute ${ }^{23}$.

Posteriormente, entre la visita de Spiegelberg en 1896 y los trabajos de Alan Gardiner y Arthur Weigall, alrededor de 1910, cuando numeran las tumbas y cierran la entrada ${ }^{24}$, algunas secciones de la escena de banquete fueron arrancadas ${ }^{25}$. Los diarios de trabajo de Norman de Garis Davies de los años 1925-1927 muestran cómo parte de los relieves del pasillo ya habían desaparecido y la pared se encontraba en un estado más próximo al actual ${ }^{26}$.

\section{Trabajo epigráfico}

La labor que se está realizando en la tumba de Hery se enfrenta a una serie de problemas que afectan al trabajo epigráfico: por un lado, el delicado estado de conservación de la decoración y la conveniencia de evitar el contacto con el original; por otro lado, el esporádico contacto visual con éste, que se reduce a las seis semanas de duración de la campaña.

La colina de Dra Abu el-Naga está formada por una piedra caliza que se caracteriza por ser muy blanda, lo que dificulta la talla del relieve, provocando que éste se fracture con mucha facilidad. A que presenta mayor dureza y que coincide con las tumbas de Djehuty y Hery, las paredes de esta última sufrieron roturas y desprendimientos poco después de la realización de su decoración y hasta es posible que durante la elaboración de la misma, principalmente en la pared este. Muchos de los fragmentos desprendidos conservaban restos del mortero que se había utilizado para ser consolidados de nuevo en su sitio, lo que nos confirma que la mala condición de la piedra obligó a que los relieves fueran constantemente recolocados desde un principio. A pesar de las restauraciones antiguas, no se pudo evitar el desprendimiento de los bloques y la pared este quedó muy dañada con el paso del tiempo. Su gran deterioro es quizá la razón por la cual todos los egiptólogos que pasaron por la tumba en el siglo XIX y comienzos del XX omitieron esta pared en sus trabajos epigráficos. En lo que respecta a los relieves del muro oeste, se encontraban en buen estado hasta la visita de Spiegelberg. La escena queda interrumpida solamente por el agujero que sirve de acceso a la tumba y que la conecta con la -399- y con la tumba de Djehuty.

El trabajo epigráfico requiere un continuo contacto con el original con el fin de trazar y colacionar los dibujos. La limitación de este contacto a la duración de la campaña obliga a buscar soluciones que permitan realizar la labor epigráfica lejos del modelo. La mejor solución está ligada a la epigrafía digital, que permite trabajar por ordenador sobre las fotografías realizadas de los relieves.

21. PM I (1): 22; Spiegelberg, 1900; Urk IV: 419-431.

22. Marquis of Northampton; Spiegelberg; Newberry, 1908.

23. Spiegelberg, Fundjournal-Theben, del 7 de noviembre de 1898 al 9 de febrero de 1899.

24. Gardiner; Weigall, 1913: 16-17.

25. No fue éste el único deterioro sufrido. Alguno de los rostros de las figuras representadas en las escenas fueron intencionadamente golpeados. Este fenómeno es muy común en Qurna y se repite en otras tumbas tebanas como en TT 31, TT 45, TT 53, TT 54, TT 128, TT 139, TT 342, etc. En 1947 se publicó un informe de inspección de la necrópolis tebana que contaba cómo algunos gafires habían confesado haber destrozado los rostros de las figuras con el fin de preservar las tumbas de los ladrones; Fakhry, 1947: 33. Sin embargo, no podemos decir que éste fuera el caso de Hery. 
La tarea principal de la Epigrafía está destinada a registrar y reproducir las inscripciones y decoración monumental con el objetivo final de hacer constar aquella información que puede ser susceptible de desaparecer y con ello contribuir a la preservación de su memoria histórica ${ }^{27}$. Esta labor puede enfrentarse con la dificultad de trabajar ante una inscripción, relieve o pintura semiborrada, destruida o saqueada y que presenta, por tanto, grandes lagunas.

La metodología epigráfica es muy amplia y variada, desde los métodos más clásicos de los Davies $^{28}$, pasando por el llevado a cabo por la Chicago House $^{29}$, hasta aquellos ligados a las últimas tecnologías $^{30}$. Con sus pros y sus contras, todos ellos son válidos a la hora de registrar la información, siempre y cuando el objetivo final de su trabajo sea salvaguardar el monumento y no dañarlo ${ }^{31}$. En la actualidad, los métodos más prácticos y respetuosos van unidos a la epigrafía digital ${ }^{32}$. Esta técnica consiste en dibujar sobre fotografías mediante programas informáticos de dibujo vectorial. Permite trabajar directamente con fotografias digitales sin requerir un contacto directo con el original, facilitando en algunos casos su conservación. La realización de copias o dibujos a través de la fotografía ofrece numerosas ventajas ${ }^{33}$ : facilita el trabajo con modelos de gran tamaño, requiere sólo contactos visuales esporádicos para colacionar, permite modificar el dibujo y corregirlo sin dificultad y su coste no es excesivamente alto. Esta metodología es la que actualmente se está utilizando en los trabajos de epigrafía de las tumbas de Djehuty y Hery.

\section{Fases de trabajo}

\section{Fase I: documentación de las paredes en su esta- do actual}

La primera fase del trabajo epigráfico consistió en fotografiar las paredes de la tumba. Sobre las fotos digitales se realizaron los dibujos en el ordenador de forma que quedara registrado el estado actual de la pared y todas sus lagunas (fig. 1). Este trabajo preliminar fue acompañado de la búsqueda de paralelos iconográficos contemporáneos a la tumba de Hery, que permitieran completar algunas de las lagunas y establecer una visión de conjunto del contenido de las diferentes escenas. Este estudio nos permitió observar cómo la temática de las escenas era muy común a comienzos de la dinastía XVIII y cómo la tumba presentaba tanto reminiscencias del estilo y de la iconografía del Reino Medio como elementos nuevos dentro del repertorio funerario ${ }^{34}$.

26. Custodiado en el archivo del Griffith Institute; Davies MSS Notebook 11.3 (1926): 1-4; Notebook 11.4 (1927): 1-3.

27. Gracias al trabajo epigráfico que realizaron Norman y Nina de Garis Davies entre 1907 y 1939 en Tebas, Amarna, Beni Hasan y Abidos, tenemos el testimonio de la decoración de algunas tumbas que en la actualidad no se han conservado. Tal ha sido el caso de la tumba de Tetiky (TT 15) en Dra Abu el-Naga, que permaneció desaparecida como consecuencia del crecimiento urbano de la zona. El derribo de las casas de Sheikh Abd el-Qurna por parte del gobierno egipcio entre los años 2006-2007 ha provocado el reciente redescubrimiento de la tumba. Davies, 1925: 10-18; Barbotin, 2008 : 151.

28. Como por ejemplo, Davies, 1920.

29. Bell, 1987.

30. Para dos estudios recientes sobre técnicas epigráficas véase Strudwick, 2001; Dorman, 2008.

31. A lo largo de la historia de la epigrafía se han utilizado algunos métodos que pudieron resultar dañinos para la piedra, en el caso del relieve. Estos métodos, como el calco, el estampado en papel mediante la presión de una esponja mojada (squeezes) o la realización de una impronta en látex exponían a la piedra a ciertos daños. Traunecker, 1987: 267-269.

32. Para los primeros trabajos que se realizaron con este tipo de técnica véase Seliger, 1995: 95-98; Manuelian, 1998.

33. La técnica de realizar dibujos sobre fotografía ya fue llevada a cabo por Tylor en El-Kab a finales del siglo XIX. Tylor, 1894; Tylor, 1900.

34. En el caso de la procesión funeraria ver, Menéndez, 2005. Un esquema de la procesión funeraria aparece en Settgast, 1963: láms. 2 y 7; sin embargo, el autor ordena los episodios de la procesión de forma errónea. 

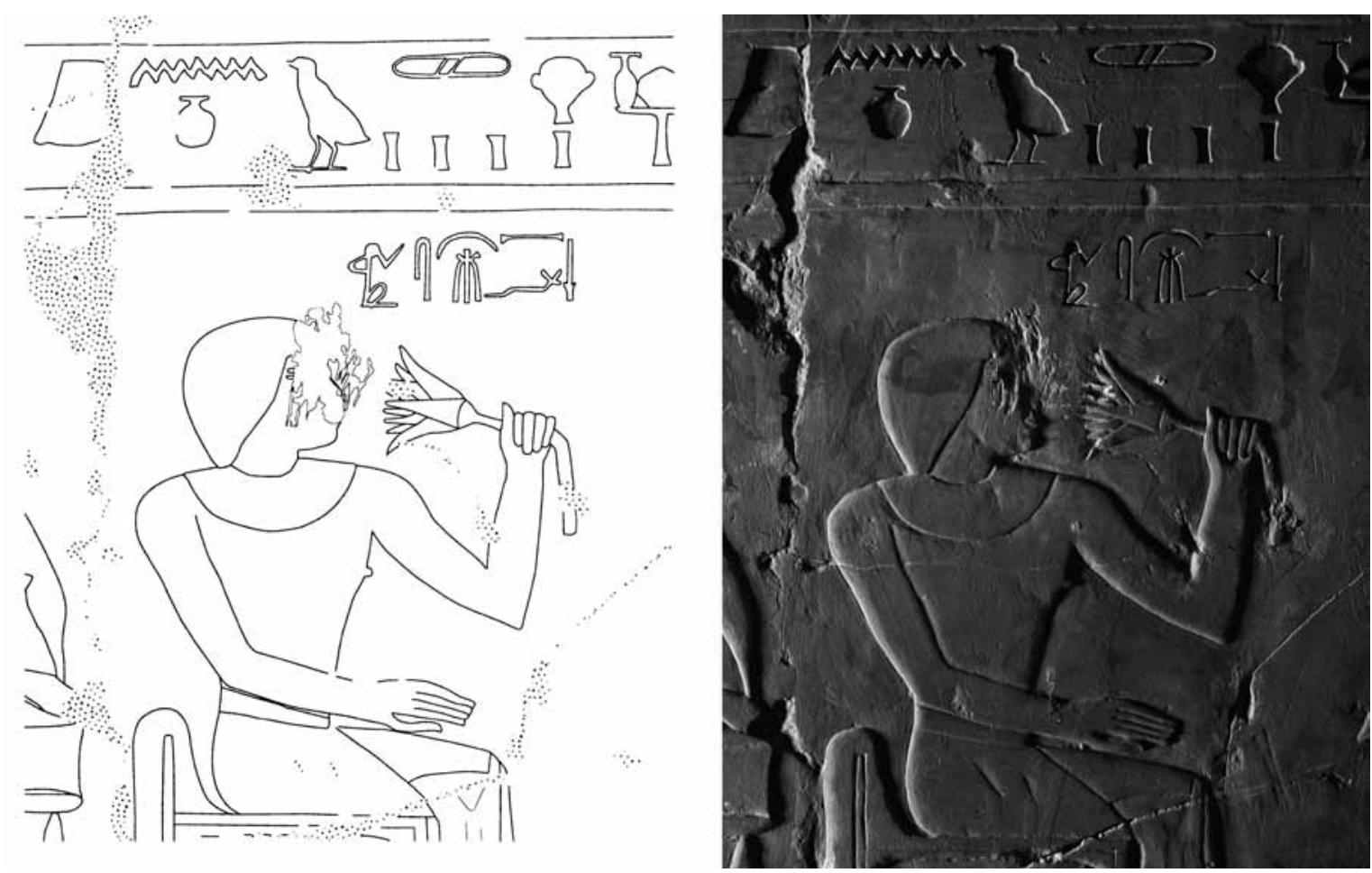

Figura 1. Detalle de la escena de banquete de la tumba de Hery. (Dibujo realizado por la autora)

Fase II: documentación de los fragmentos de relieve desprendidos de las paredes.

Los trabajos de limpieza del interior de las tumbas y la excavación del exterior para sacar a la luz los patios de entrada, dieron como fruto el hallazgo de un gran número de fragmentos de relieve procedentes del interior de la TT 11 y TT 12, así como de otras tumbas de los alrededores. Durante [ 114 ] los ocho primeros años de trabajo de campo se han encontrado un total de 1.684 fragmentos de relieve, de los cuales alrededor de 250 procedían de la tumba de Hery. Los relieves que fueron hallados en el exterior posiblemente procedían de desprendimientos antiguos. Como hemos mencionado anteriormente, la caliza es demasiado blanda en algunos sectores y eso provocó la caída de muchos fragmentos y su posterior recolocación ya en época antigua. Estos fragmentos restaurados debieron de ser los primeros que cayeron y quizás los que acabaron en el exterior en los diferentes procesos de limpieza de la tumba.

Los relieves que se han encontrado hasta el momento pertenecen principalmente a tres categorías dentro del repertorio iconográfico tebano:

a. Frisos heker, empleados para encuadrar las escenas.

b. Relieves procedentes de las escenas de caza y pesca en los pantanos de la pared este.

c. Relieves procedentes de la procesión funeraria de la pared oeste.

Destaca la falta de fragmentos correspondientes a otras escenas, como los relacionados con la peregrinación a Abidos, la procesión de ofrendas, el banquete, etc. 
El procedimiento seguido con las piezas comenzó por la usual catalogación y siglado. Posteriormente, se fotografiaron y se realizaron los dibujos vectoriales de las superficies talladas (fig. 2). El trabajo por ordenador permite además, "jugar" con aquellos fragmentos de relieve que se pueden reubicar dentro de la escena, o bien juntar los que encajaban entre sí (figs. 3 y 4).

\section{Fase III: Squeezes de Spiegelberg}

La última fase de nuestro trabajo epigráfico se centra en los squeezes o estampados en papel que realizó Spiegelberg en 1896. Consisten en pliegos de papel de un tamaño aproximado de 80 x $120 \mathrm{~cm}$, con una textura gruesa y porosa, como la del papel que se utiliza para pintar acuarelas. Se colocaban en la pared y eran presionados con una esponja mojada, de forma que se iban reblandeciendo y adaptándose al relieve. Luego, al secarse de nuevo, quedaba grabada en tres dimensiones la forma del relieve con todo detalle. Aunque este método epigráfico resultaba dañino para la pared y para la pintura que ésta pudiera conservar, hoy en día constituye un importante documento para el estudio de las tumbas. Actualmente uno de los trabajos que se está realizando dentro del "Proyecto Djehuty" está ligado al estudio y publicación de estos squeezes, que se conservan en el Griffith Institute de Oxford, donde han permanecido desde el 27 de julio de $1926^{35}$. Gracias a la colaboración del Dr. Jaromir Malek, conservador del archivo, los calcos fueron fotografiados, ya que su tamaño dificultaba que fueran escaneados. Esta labor fue muy similar a la que se sigue a la hora de fotografiar relieves o grabados, pues se necesitó una luz rasante que destacara las formas. Además, se documentó tanto el anverso como el reverso, ya que en oca- siones se veían mucho mejor los detalles en este último. En algunos casos, el reverso aún conserva restos de barro y pintura de los grafitos realizados sobre los relieves, y la suciedad resultó ser una ayuda a la hora de definir las formas.

Una vez fotografiados, de nuevo la epigrafia digital fue de gran ayuda. Se dibujaron los calcos por separado y posteriormente se procedió al montaje de los dibujos con el fin de obtener una visión completa del estado de la pared en 1896, momento en el que fueron realizados los squeezes (fig. 5). El resultado de la combinación de estos trabajos epigráficos permite completar las lagunas actuales de la pared, de especial importancia para una futura restauración.

En primer lugar, el estudio de paralelos contemporáneos permite conocer la escena completa. El estudio de éstos permite reubicar muchos de los fragmentos que se han desprendido y que se han encontrado en los trabajos de excavación en el exterior de la tumba. Por último, los squeezes de Spiegelberg permiten documentar los fragmentos que fueron saqueados de la pared y cuyo paradero se desconoce, además de observar los cambios producidos en los últimos cien años (fig. 6).

\section{Conclusión}

La misión hispano-egipcia encargada de las tumbas de Djehuty (TT 11) y Hery (TT 12) tuvo desde el primer momento el objetivo de estudiar y documentar las inscripciones y relieves que las componían. La labor epigráfica llevada a cabo durante estos años ha permitido llegar a las siguientes conclusiones.

El estudio de paralelos junto con la historiografía epigráfica es de gran utilidad para reconstruir escenas

35. Los squeezes iban acompañados de una nota firmada por Spiegelberg que informaba que Jean Capart los había tenido en préstamo en los Musées Royaux d'Art et d'Histoire de Bruselas desde septiembre de 1910. Spiegelberg expresa en la nota su deseo de que éstos fueran publicados por la Egypt Exploration Society, pero no fue así. 
dañadas por el paso del tiempo. El hallazgo de los squeezes de Spiegelberg en el Griffith Institute es una invitación a la búsqueda historiográfica en los archivos, la cual puede aportarnos información que hasta ahora considerábamos definitivamente perdida.

El uso de la epigrafia digital permite la realización de los dibujos de las inscripciones e incluso de aque- llos objetos con los que se mantiene un contacto esporádico. De la misma forma, el dibujo sobre fotografías ayuda a la mejor conservación de los monumentos y objetos, sobre todo si tienen restos de pintura. La restauración final necesitará la fusión de todos estos procesos de trabajo para completar, en la medida de lo posible, la decoración e inscripciones de la pared. 

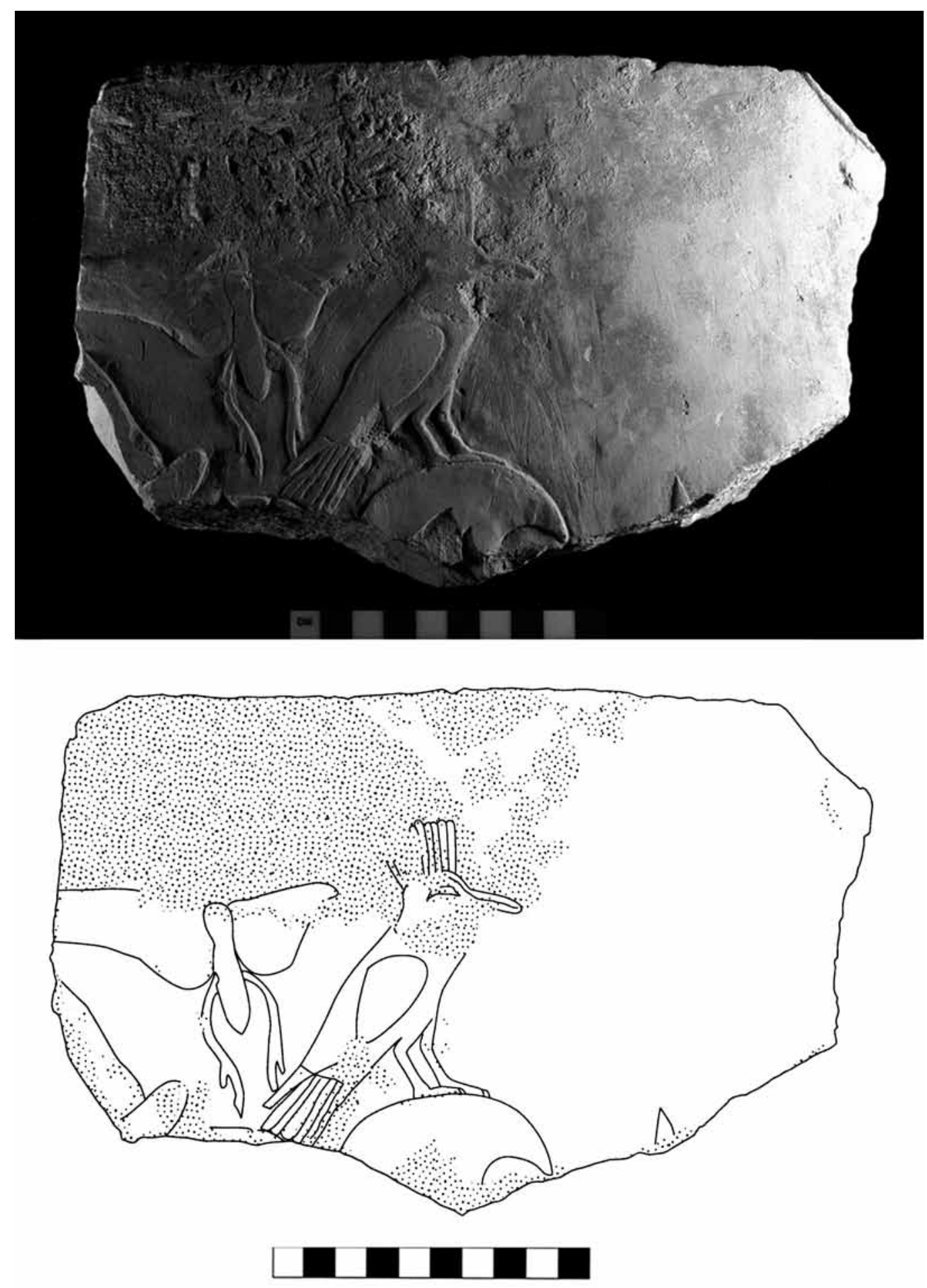

Figura 2. Fragmento de relieve procedente de la escena de caza y pesca en los pantanos de la pared este de la tumba. (Dibujo realizado por la autora) 

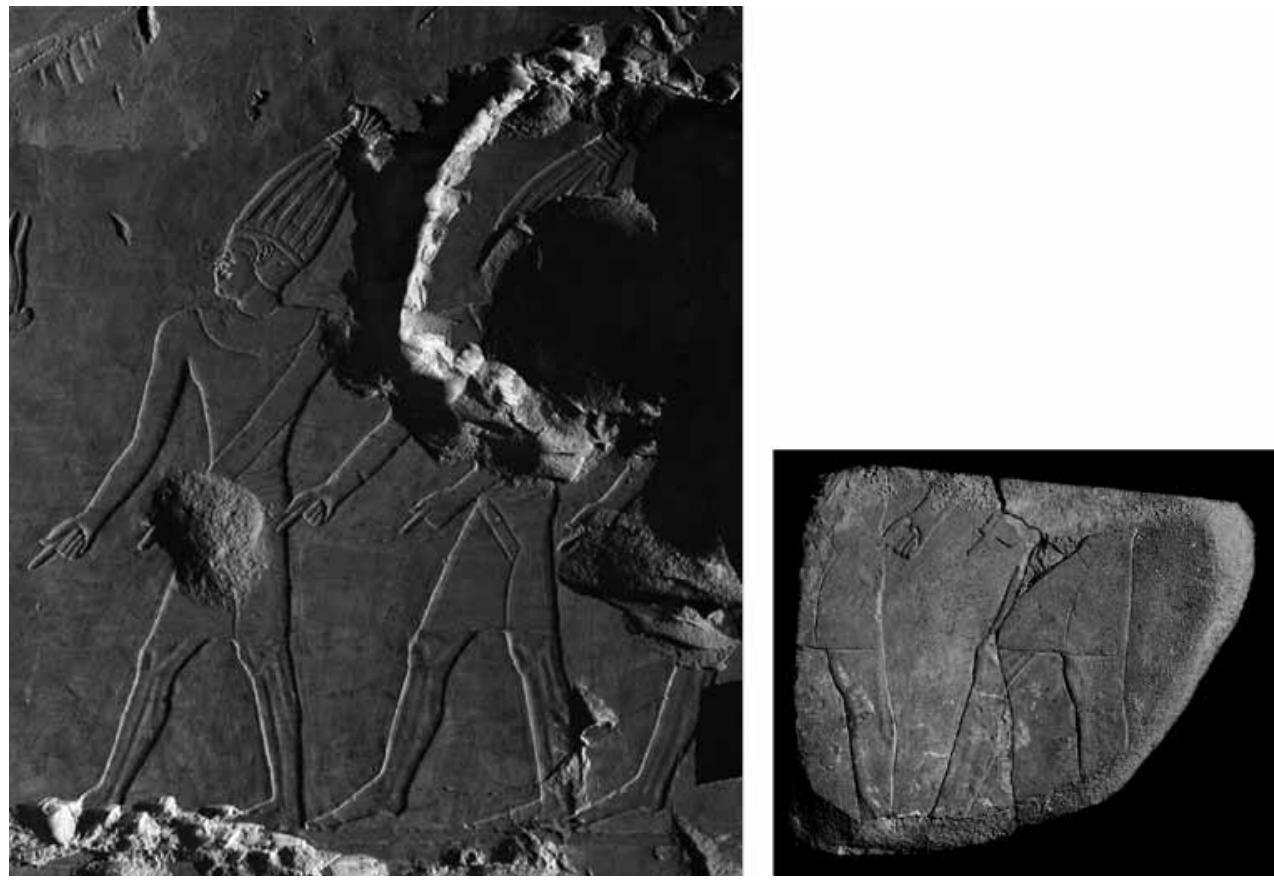

Figura 3. Fragmento de relieve hallado en la excavación del exterior perteneciente a la escena de la danza Mww en la procesión funeraria

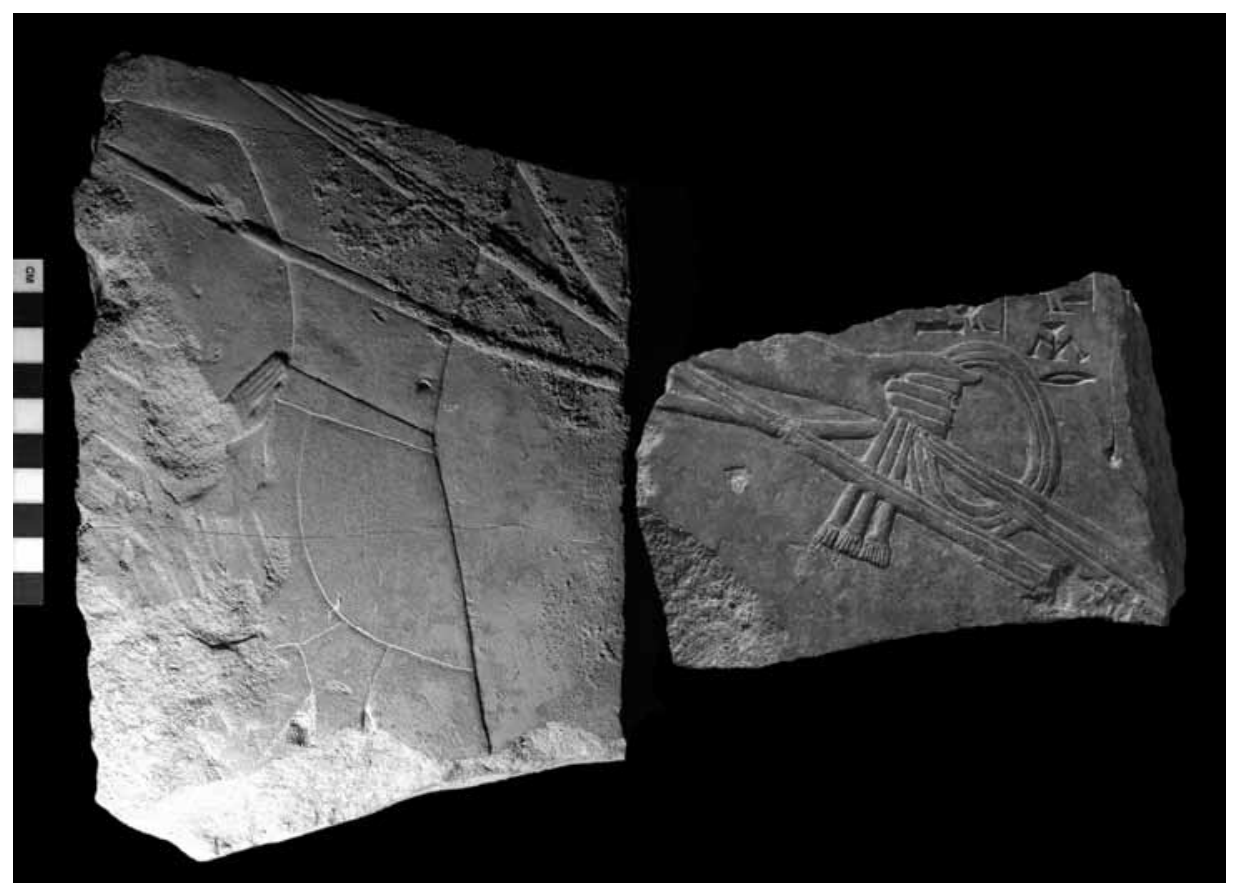

Figura 4. Fragmentos de relieve pertenecientes a la escena de caza y pesca. El fragmento de la izquierda fue hallado en el suelo del pasillo de la tumba, mientras que el de la derecha procede de la excavación del exterior 

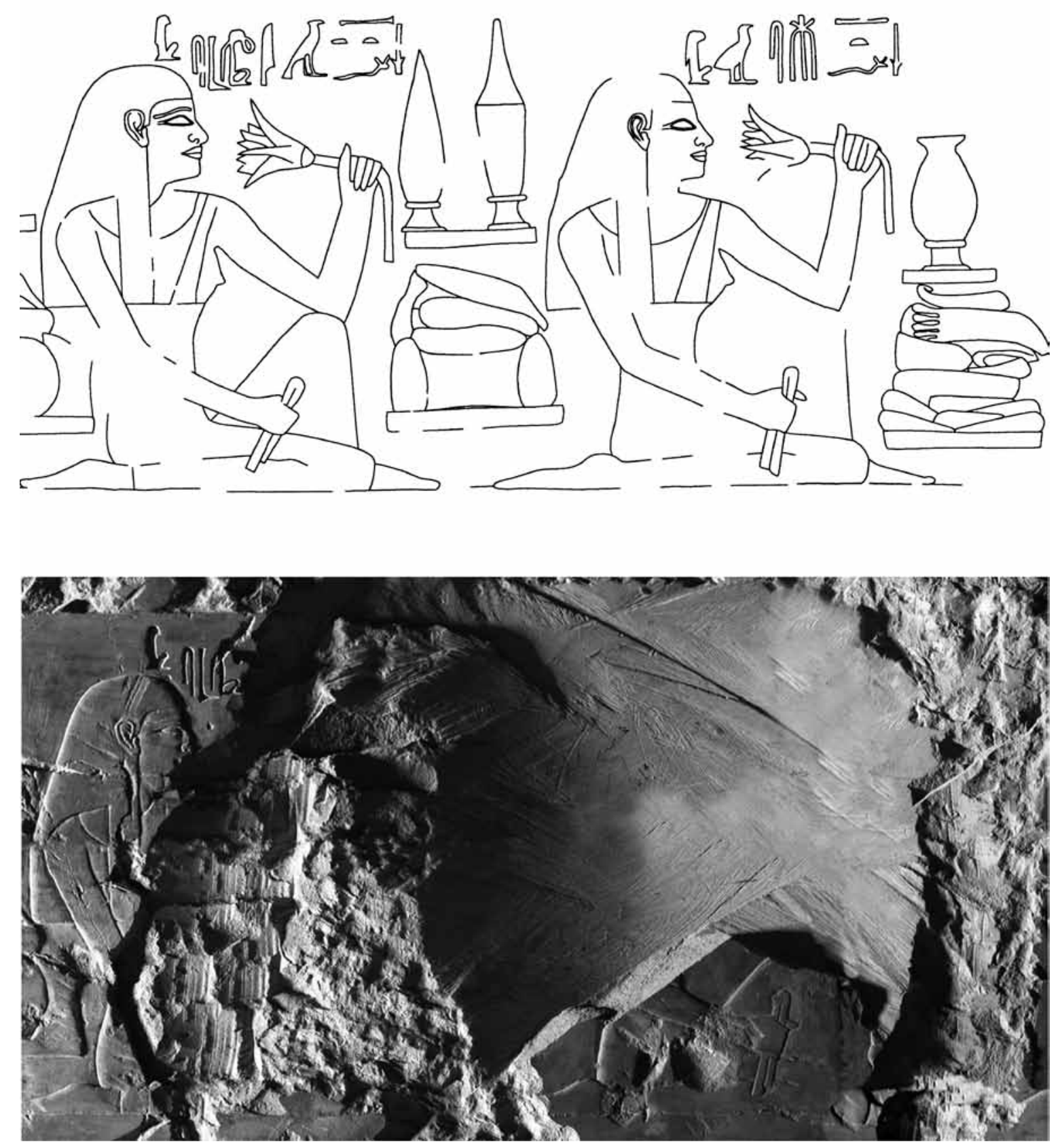

Figura 5. Superior. Dibujo digital del banquete realizado sobre los squeezes de Spiegelberg. Inferior. El mismo detalle en la actualidad. Al compararlos observamos algunos de los daños sufridos por los relieves de la tumba desde 1896. (Dibujo realizado por la autora) 


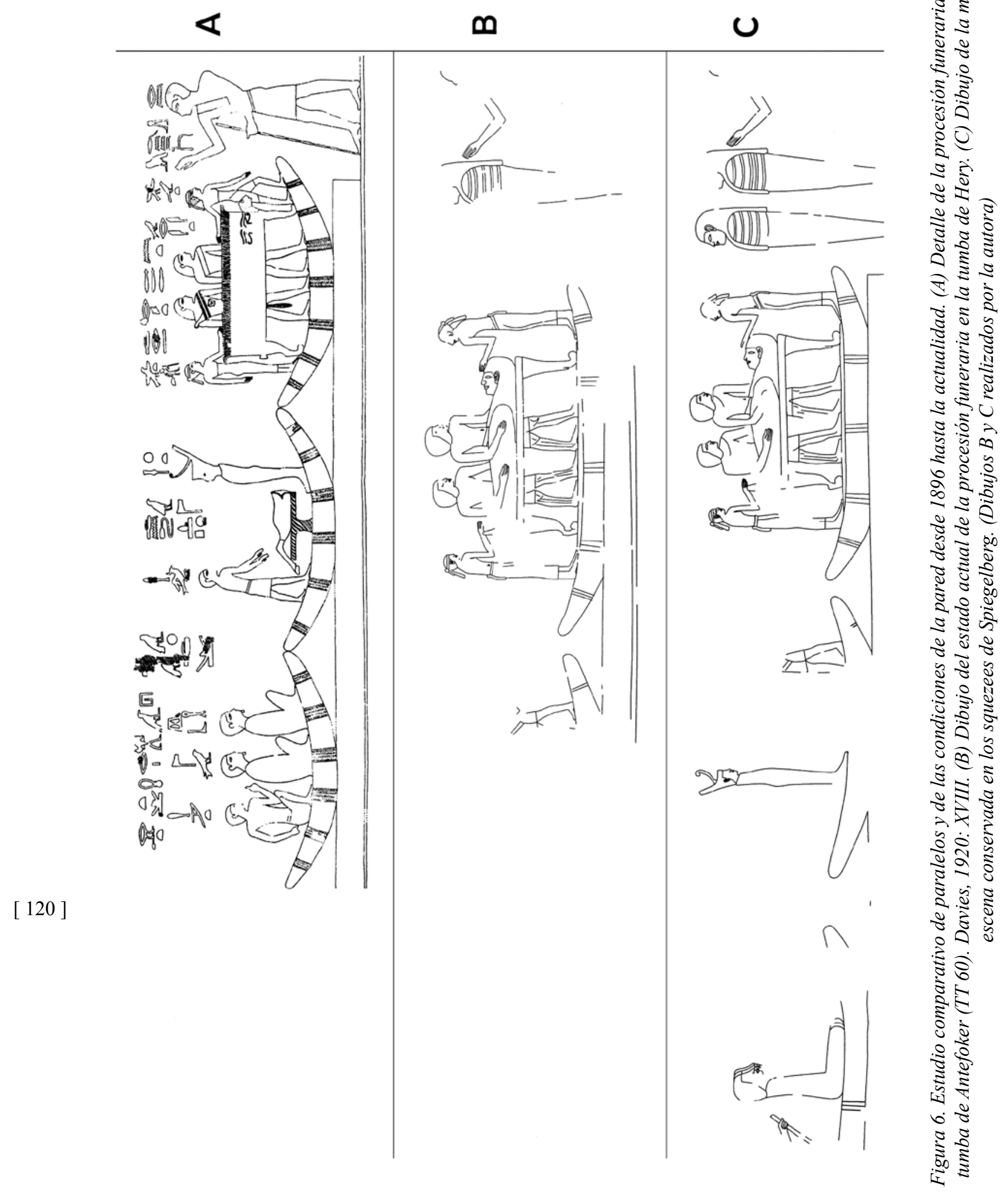




\section{Bibliografía}

BARBotin, $\mathrm{CH}$.

2008 Âhmosis et le début de la XVIIIe Dynastie. Paris.

BELL, L.

1987 The Epigraphic Survey: the philosophy of Egyptian epigraphy after sixty years' practical experience, en Assmann, J.; Burkard, G. y Davies, V. (eds.): Problems and Priorities in Egyptian Archaeology, London: 43-55.

BOHLEKE, B.

2000 The Overseers of Double Granaries of Upper and Lower Egypt in the Egyptian New Kingdom, 1570-1085 B. C. Ann Arbor, Michigan.

DAVIES, N. DE G.

1920 The Tomb of Antefoker, vizier of Sesostris I, and his wife, Senet (No. 60). London.

1925 The Tomb of Tetaky at Thebes (No. 15), JEA 11: 10-18.

DEWATCHER, M.

1985 Nouvelles informations relatives à la exploitation de la nécropole royale de Dra Abou Neggah, RdE 36: 43-66.

Dorman, P. F.

2008 Epigraphy and Recording, en Wilkinson, R. H. (ed.): Egyptology Today, Cambridge: 77-97.

Champollion, J.-F.

1873 Notices Descriptives. Genève.

FAKHRY, A.

1947 A Report on the Inspectorate of Upper Egypt, $A S A E$ 46: 25-54.
GaLÁN, J. M.

2006 The Tombs of Djehuty and Hery (TT 1112) at Dra Abu el-Naga, en Goyon, J.-Cl. y Cardin, C. (eds.): IXe Congrès International des Egyptologues, (OLA 150), Leuven: 779-789.

Gardiner, A. H.; Weigall, A. E. P.

1913 A Topographical Catalogue of the Private Tombs of Thebes. London.

KAMPP, F.

1996 Die thebanische Nekropole: zum Wandel des Grabgedankens von der XVIII bis zur XX. Dynastie. (Theben 13). Mainz am Rhein.

LePsiUs, $\mathrm{K}$.

1897-1913 Denkmäler aus Aegypten und Aethiopien. Text. Vol. III. Berlin.

MANUELIAN, P. DER

1998 Digital Epigraphy: An Approach to Streamlining Egyptological Epigraphic Method, JARCE 35: 97-113.

Marquis of Northampton; SpiegelberG, W.;

NEWBERRY, P. E.

1908 Report on some Excavations in the Theban Necropolis during the Winter of 1898 1899. London.

MENÉNDEZ, G.

2005 La procesión funeraria de la tumba de Hery (TT 12) en Dra Abu el-Naga, BAEDE 15: 29-65.

Nims, C. F.

1980 The Tomb of Kheruef. Theban Tomb 192. (OIP 102). Chicago. 
Romano, J.

1976 Observations on Early Eighteenth Dynasty Royal Sculpture, JARCE 13: 97111.

1983 A Relief of King Ahmose and Early Eighteenth Dynasty Archaism, BES 5: 103-115.

Roth, A. M.

1999 The Absent Spouse: Patterns and Taboos in Egyptian Tomb Decoration, JARCE 36: 37-53.

Seliger, F.

1995 Vorstellung von zwei Computer-Programmen. I: Das DASS-Project. II: Eine Grabungs-Dokumentation, GM 114: 8499.

Serrano Delgado, J. M.

2005 Baki, supervisor del ganado de Amón, $B A E D E$ 15: 85-98.

Settgast, J.

1963 Untersuchungen zu Altägyptischen Bestattungsdarstellungen. Glückstadt / Hamburg / New York.

SPIEGELBERG, W.

1900 Die Northampton Stele, RecTrav 22: 115-125.
Strudwick, N.

2001 Problems of recording and publication of paintings in the Private Tombs of Thebes, en Davies, W. V. (ed.): Colour and Painting in Ancient Egypt, London: 126-140.

2003 Some aspects of the archaeology of the Theban Necropolis in the Ptolemaic and Roman periods, en Strudwick; N. y Taylor, J. H. (eds.): The Theban Necropolis. Past, Present and Future, London: 167-188.

TRAUNECKER, C.

1987 Les Techniques d'épigraphie de terrain: principes et pratique, en: Assmann, J.; Burkard, G. y Davies, V. (eds.): Problems and Priorities in Egyptian Archaeology, London: 261-298.

TYLOR, J. J.

1894 The Tomb of Paheri. London.

1900 The Tomb of Renni. London.

Whale, S.

1989 The Family in the Eighteenth Dynasty of Egypt. A Study of the Representation of the Family in Private Tombs. (The Australian Centre for Egyptology Studies 1). Sidney.

WINLOCK, H. E.

1924 The Tombs of the Kings of the Seventeenth Dynasty at Thebes, JEA 10: 217-277. 


\section{Trabajos de Egiptología Papers on Ancient Egypt}

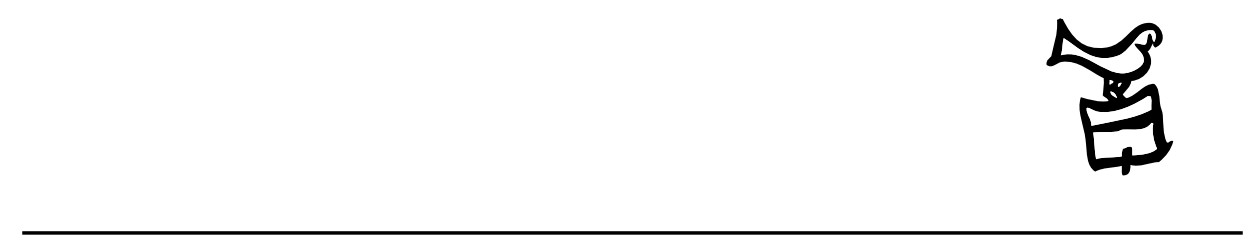

Número 5/2 2009 


\section{Actas \\ III Congreso Ibérico de Egiptología III Congresso Ibérico de Egiptologia}

Editores

Miguel Ángel Molinero Polo Covadonga Sevilla Cueva 


\title{
Editor
}

Miguel Ángel Molinero Polo

Universidad de La Laguna

\section{Consejo Editorial}

\author{
Antonio Pérez Largacha \\ Universidad de Castilla-La Mancha \\ José-R. Pérez-Accino \\ Birkbeck, Universidad de Londres \\ Covadonga Sevilla Cueva \\ Universidad Autónoma de Madrid
}

\section{Comité Científico}

Josep Cervelló i Autuori

Universitat Autònoma de Barcelona

$\mathrm{M}^{\mathrm{a}}$ José lópez Grande

Universidad Autónoma de Madrid

Josep Padró i Parcerisa

Universitat de Barcelona

$\mathrm{M}^{\mathrm{a}}$ Carmen Pérez Die

Museo Arqueológico Nacional, Madrid

Ester Pons Mellado

Museo Arqueológico Nacional, Madrid

José M. Serrano Delgado

Universidad de Sevilla

\section{Colaboradores Editoriales}

Linda Steynor

English editorial assistant

Hervé Mouriacoux

Assistant éditorial pour la langue française 
Trabajos de Egiptología está producida por Isfet. Egiptología e Historia c/ Blanco $1,2^{\circ}$

38400 Puerto de la Cruz

Tenerife-Islas Canarias

España

Maquetación: Proyecto Limón

(C) Autores de los artículos aparecidos

y Consejo Editorial de Trabajos de Egiptología - Papers on ancient Egypt

Depósito Legal: TF-2303-2009

ISSN: $1695-4750$

Imprime: Gráfica Los Majuelos, S.L.L.

imprenta@graficaslosmajuelos.com

Tfno.: 922311455 


\section{Comité Científico \\ III Congreso Ibérico de Egiptología III Congresso Ibérico de Egiptologia}

Miguel Á. Molinero Polo

Universidad de La Laguna

Presidente del Comité Organizador del III Congreso Ibérico de Egiptología

Miembro del Comité Organizador del I Encuentro de Egiptología

Josep Cervelló Autuori

Universitat Autònoma de Barcelona

Presidente del Comité Organizador del II Congreso Ibérico de Egiptologia

José Manuel Galán Allué

Consejo Superior de Investigaciones Cientificas

Director del Proyecto Djehuty, Luxor, Egipto

$\mathrm{M}^{\mathrm{a}}$ Helena Trindade Lopes

Universidad de Lisboa

Directora de la Misión Arqueológica Portuguesa en Menfis

Josep Padró i Parcerisa

Universitat de Barcelona

Director de la Misión Arqueológica de Oxirrinco

Antonio Pérez Largacha

Universidad de Castilla - La Mancha

Miembro del Comité Organizador del I Encuentro de Egiptología

José Ramón Pérez-Accino

Birkbeck College, University of London

Miembro del Comité Organizador del I Encuentro de Egiptología

$\mathrm{M}^{\mathrm{a}}$. Carmen Pérez Díe

Museo Arqueológico Nacional

Directora de la Misión Arqueológica Española en Heracleópolis Magna, Egipto

Covadonga Sevilla Cueva

Universidad Autónoma de Madrid

Miembro del Comité Organizador del I Encuentro de Egiptología 